\title{
Special issue on evolutionary multi-objective optimization (EMO): theory and applications
}

\author{
Zhihua Cui ${ }^{1} \cdot$ Xiao-Zhi Gao ${ }^{2}$
}

Published online: 7 June 2019

๑) Springer-Verlag GmbH Germany, part of Springer Nature 2019

Evolutionary Multi-objective Optimization (EMO) is a collection of the state-of-the-art theoretical research, design challenges, and applications in the field of multi-objective optimization paradigms using evolutionary algorithms. It is concerned with mathematical optimization problems involving more than one objective function to be optimized simultaneously. The EMO has been applied in many fields of science, including engineering, economics, and logistics, where the optimal decisions need to be made in the presence of the trade-offs between two or even more conflicting objectives. We believe that the series of works in this special issue provide a useful reference for learning the current progresses on the EMO. In total, six papers have been selected to reflect the call of the thematic vision. The contents of these studies are briefly described as follows.

In the paper, 'Evolutionary dynamic multi-objective optimization algorithm based on Borda count method', MaysamOrouskhani, Mohammad Teshnehlab, and Mohammad Ali Nekoui propose a novel dynamic multi-objective optimization algorithm, which is composed of three parts: change detection, response to change, and optimization process. The first step is to use Sentry solutions to detect the environmental change and to advise the algorithm when a change occurs. Next, to increase the diversity of solutions, the worst solutions should be selected and removed from population and re-initialized with new solutions. The main idea is to use Borda count method, an optimal rank aggregation technique ranking the solutions in order of preference and nominating

Zhihua Cui

cuizhihua@tyust.edu.cn

Xiao-Zhi Gao

xiao-zhi.gao@uef.fi

1 Complex System and Computational Intelligence Laboratory, Taiyuan University of Science and Technology, Taiyuan, China

2 School of Computing, University of Eastern Finland, Kuopio, Finland the worst solutions that should be removed. The last step is the optimization process using the multi-objective Cat Swarm Optimization (CSO). The CSO utilizes the population that has been improved from the previous step to estimate the best solutions, and can converge to the optimal Pareto front. The performance of the proposed algorithm is tested on the dynamic multi-objective benchmarks, and the simulation results are compared with the ones achieved by conventional algorithms. The results indicate that it can effectively track the time-varying optimal Pareto front and achieve competitive results.

It is difficult to maintain the balance between convergence and diversity for Many-objective Optimization Problems (MaOPs) in the EMO algorithms, which are useful technologies to solve the Multi-objective Optimization Problems (MOPs). However, with larger optimization objectives, enough Pareto selection pressure can be loosed, and the performances of the algorithms are significantly reduced. The decomposition-based EMO developed for the MaOPs have been shown to be effective. In the paper, 'An improved biogeography/complex algorithm based on decomposition for many-objective optimization', C. Wang et al. propose a hybrid decomposition-based $\mathrm{BBO} /$ Complex algorithm (HDB/BBO) for the MaOPs. Firstly, a set of uniformly distributed weight vectors and K-means aggregate method are introduced for decomposing the MaOPs into several subsystems. Secondly, the inferior migrated islands will not be chosen unless they pass the Metropolis criterion twice during the within-subsystem migration and cross-subsystem migration. The Penalty-based Boundary Intersection (PBI) distance is used to calculate the neighbor islands distance for balancing the algorithm of convergence and diversity. Finally, after mutation and clear duplication, a uniform distribution Pareto set can be obtained. Experimental results on both the DTLZ and WFG benchmarks problems demonstrate the superiority of the proposed algorithm in comparison with three state-of-the-art algorithms in terms of both convergence and diversity. 
The public opinion synchronization on the network platform is becoming one of the most important issues worthy of a careful study. In the paper, 'Modeling and intelligent optimization of social collective behavior with online public opinion synchronization', R. Xiao, J. Li, and T. Chen take the synchronization evolution phenomenon as an objective and adopt the Artificial Bee Colony (ABC) to evaluate the network synchronization effects with optimization theory to find out an appropriate network structure. Firstly, they use the Kuramoto oscillators as a metaphor of the social system collective behavior. Secondly, combined with the social network characteristics obtained from the data of Sina MicroBlog, a synchronization evolution model of Internet public opinion based on the Kuramoto one is established. Subsequently, the EMO model is built up, and the ABC method is applied to optimize the level of the network synchronization, the synchronization starting time, and the cost of public opinion synchronization. Finally, the case analysis on the "Double Eleven" Internet marketing as well as Cadmium poisoned rice event demonstrates that the synchronization performance of weakly coupled system can be enhanced by offering a reasonable configuration of the connection cost and the synchronization duration cost. In addition, a certain degree of increase in the input cost can promote the synchronization performance and extend the synchronization duration significantly.

To study the general analytical model for the optimization of the trade-off between the stability and energy functions for biped humanoid robots is very tedious job. In the paper, 'Multiobjective optimized bipedal locomotion', Manish Raj, Vijay BhaskarSemwal, and GC Nandi present a novel analytical method to develop the multiobjective function including the energy and stability functions. The challenge is to develop the analytical model for stability and energy for the Single Support Phase (SSP), Double Support Phase (DSP), and the transition to SSP-DSP and vice versa. The energy function has been developed by a unique approach of orbital energy concept and the stability function obtained by modifying the pre-existing Zero Moment Point (ZMP) trajectory (the trajectories generated by the mathematical equations of the ZMP). These functions are optimized using real coded genetic algorithm to produce an optimum set of walk parameters. The analytical results show that, when the energy function is optimized, the stability of the robot decreases. Similarly, if the stability function is optimized, the energy consumed by the robot increases. Thus, there is a clear trade-off between the stability and energy functions. The EMOalgorithm is used to obtain the optimum value of the walk parameters. The results are verified by the $\mathrm{NaO}$ robot. This approach increases the energy efficiency of $\mathrm{NaO}$ robot by $67.05 \%$ and stability increases by $75 \%$, which can be utilized on all the ZMP classed bipeds(HOAP, Honda robots).
The Proportional-Integral-Derivative (PID) controller continues to provide the easiest and most effective solutions to most of the industrial applications in recent years. However, it is often poorly tuned and complicated in practice compared to other tuning methods. In the paper, 'Tuning of a PID controller using evolutionary multi objective optimization methodologies and application to the pulp and paper industry', K. Nisi, B. Nagaraj, and A. Agalya present a new multiobjective optimization approach involving the genetic algorithms, evolutionary programming, particle swarm optimization, and bacterial foraging optimization. Their multiobjective optimization algorithm is used to tune the PID controller parameters, and the performances yielded have been compared with the conventional methodologies like Ziegler Nichols method. The results illustrate that the use of the multiobjective optimization approach-based controller tuning can improve the performances of the processes with regard to the time domain specifications and performance indexes, set point tracking, and regulatory changes. This paper also describes various multiobjective optimization algorithms with implementation to tune the PID controller used in the real time paper machine DCS of the pulp and paper industry processes.

Insertion of Distributed Generation (DG) in the existing distribution systems has effectively improved its performance and operation. Many different methods for the planning of the distribution systems with the DG insertion are presented by researchers. In the paper, 'A multi objective approach for placement of multiple DGs in the radial distribution system' (authored by Snigdha R. Behera and B. K. Panigrahi), a multiobjective approach has been proposed to maximize the mutual benefits of both the distribution system operator and DG owner. The contradictory relationship between the reduction in the MVA rating of the DGs and reduction of the power losses of the system is the motivation for this multi-objective approach. The best compromised size of the DGs in the MVA and their operating power factors and positions are obtained to reduce the system active power loss along with the reduction of the DGs size. The 69-bus and 85-bus radial distribution system are considered as the test systems here. The Pareto-front of non-dominated solutions is obtained by using the Multi-Objective Differential Evolution (MODE) optimization algorithm. The performance of the MODE is compared with that of the MultiObjective Particle Swarm Optimization (MOPSO). Different system operating indices, such as active power loss, reactive power loss, and voltage deviation, are evaluated to show the effects of the best compromised solution of DGs placement on distribution system.

This special issue on 'Evolutionary Multi-objective Optimization (EMO): Theory and Applications' presents the latest research and development in evolutionary multi-objective optimization. The guest editors expect that the readers will 
benefit from the papers presented in the issue. The guest editors of this special issue would like to thank all the authors for submitting their interesting work. We are grateful to the reviewers for their great contributions to the special issue. Moreover, the guest editors are very much grateful to the Editor-in-Chief of the International Journal of Machine Learning and Cybernetics, Prof. Xizhao Wang, and all the editorial teams at Springer for the assistance during the paper submission, review, and production steps. Zhihua Cui's work is supported by the National Natural Science Foundation of
China under Grant No. 61806138, Xiao-Zhi Gao's work was partially supported by the National Natural Science Foundation of China (NSFC) under Grant 51875113.

Publisher's Note Springer Nature remains neutral with regard to jurisdictional claims in published maps and institutional affiliations. 